\title{
A statistical study on solar soft X-ray flare and the sources
}

\author{
Juan Guo \\ Key Laboratory of Solar Activity, National Astronomical Observatories, Chinese Academy of \\ Science \\ email: guojuan@bao.ac.cn
}

\begin{abstract}
We surveyed 28 year of Solar X-ray flares and sunspot group records supplied by the National Geophysical Data Center (NGDC) to study the dependence of different class Xray flares on the magnetic structure of sunspot groups statistically. It is found that $82.5 \% \mathrm{X}$-class flare, $51.4 \%$ M-class flare, $26.3 \%$ C-class flare, and 10.1\% B-class flare occurred in the sunspot group with $\beta \gamma \delta$ structure. There are $6.6 \%$ X-class flares, $17.5 \%$ M-class flare, $39.1 \%$ C-class flare, and $60.4 \%$ B-class flare occurred out of sunspot group.
\end{abstract}

Keywords. flares, magnetic fields

\section{Introduction and Data}

In 1960, Kunzel (1960) pointed out the first clear connection between flare productivity and magnetic structure. He introduced a new magnetic classification, $\delta$, to supplement Hale's $\alpha, \beta$, and $\gamma$ classes. The delta region included a penumbra enclosing umbrae of both positive and negative polarity. Although Kunzel's statistical evidence was weak, time has proven his conclusions correct, and the delta configuration became the critical ingredient to the solar flare problem, widely used by forecasters.

Using 8 year of active regions, Sammis et al. (2000) studied the dependence of large flares occurrence on the magnetic structure of sunspots. They found that there is a general trend for large regions to produce large flares, but it is less significant than the dependence on magnetic class. They confirmed the close correlation between large flare and delta sunspots.

In the present work, we use 28 year of Solar X-ray flares and sunspot group records supplied by the National Geophysical Data Center (NGDC) from 1983 April to 2001 December. For the sunspot group, we use the USAF sunspot record in the same duration. We define the complexity of magnetic field of sunspot from low to high order as: $\alpha, \beta$, $\gamma, \beta \delta, \gamma \delta, \beta \gamma \delta$. For each sunspot, we use the highest magnetic classification. Thus, a sunspot is considered the highest level if it reached that value once in its disk passage. The effects of returning spot groups are ignored in this study.

We collect a dataset which list each flare and the region in which it occurred, if known, along with the magnetic classification, area, and position.

\section{Result}

Table 1 shows the percentage of different class flares occurred in sunspot of different magnetic type, when taking all the flares in 22 and 23 solar cycles in consideration. It is found that $82.5 \% \mathrm{X}$-class flare and $51.4 \%$ M-class flare occurred in $\beta \gamma \delta$ type sunspot groups. However, there are $39.1 \%$ C-class flares occurred out of sunspot groups, and only $26.3 \%$ C-class flare occurred in the sunspot group with $\beta \gamma \delta$ structure. There are $60.4 \%$ 
Table 1. Percentage of different class flares occurred in different source regions.

\begin{tabular}{|c|c|c|c|}
\hline $\mid$ flare class & $\mathrm{P}_{n o A R}\left|\mathrm{P}_{\alpha}\right|$ & $\mathrm{P}_{\beta}\left|\mathrm{P}_{\gamma}\right|$ & $\mathrm{P}_{\beta \delta}\left|\mathrm{P}_{\gamma \delta}\right| \mathrm{P}_{\beta \gamma \delta}$ \\
\hline $\mathrm{X}$ & $6.6|0.0|$ & \begin{tabular}{l|l|} 
& 6.9
\end{tabular} & $2.3|0.3| 82.5$ \\
\hline M & $17.5|0.0|$ & $8.9|16.4|$ & \begin{tabular}{l|l|l|l}
5.5 & 0.4 & 51.4
\end{tabular} \\
\hline $\mathrm{C}$ & $39.1|0.1|$ & $12.8|16.9|$ & $4.3|0.5| 26.3$ \\
\hline B & $60.4|0.3|$ & $12.2 \mid 14.1$ & $2.7|0.1| 10.1$ \\
\hline
\end{tabular}

B-class flares occurred out of sunspot groups, and only 10.1\% B-class flare occurred in the sunspot group with $\beta \gamma \delta$ structure.

\section{Discussion}

In the present work, we surveyed 28 years of X-flare and sunspot record to study the dependence of the different class flares on the magnetic field type of sunspot.

For the flares occurred without sunspot group records, we examined the SGD weekly reports and found that still many X-class flares were describe to be likely occurred in some complex sunspots groups which will appeared in and rotate out the solar disk.

Note only use the data from the USAF stations and omit to use the Mount Wilson record because that Mount Wilson treats delta as a separate class, counting any region with two opposite polarities in a single penumbra, even if these are not the main spots. If consider the Mount Wilson record in, it is found that $90.7 \%$ X-class flare, $66.4 \%$ M-class flare, $37.5 \%$ C-class flare, and 15\% B-class flare occurred in the sunspot group with $\beta \gamma \delta$ structure.

\section{References}

Kunzel H. 1960, Astron. Nachr, 285, 271

Sammis I., Tang F., \& Zirin H. 2000, ApJ, 540, 583 